\title{
Image Constrast Control based on Łukasiewicz's Operators
}

\author{
N.M. Hussein Hassan \\ Instituto de Microelectrónica de Sevilla (CNM-CSIC) \\ c/ Americo Vespucio s/n. 41092-Sevilla, Spain
}

\author{
A. Barriga \\ Instituto de Microelectrónica de Sevilla (CNM- \\ CSIC)/Universiy of Seville \\ c/ Americo Vespucio s/n. 41092-Sevilla, Spain
}

\begin{abstract}
This paper describes a technique to control the contrast in images based on the application of Lukasiewicz algebra operators. In particular, the technique is based on the bounded-sum and the bounded-product. An interesting feature when applying these operators is that it allows low cost hardware realizations (in terms of resources) and high processing speed.
\end{abstract}

\section{INTRODUCTION}

The sensory human systems are organized to respond rapidly to the temporary and spatial changes of the energy stimulus. When there is a temporary change in the energy applied to the sensor there is initially a strong response. Then the senses adapt rapidly (they respond less) to the constant and continued use of the energy. The visual system shows two types of behaviors: firstly to have the aptitude to answer (to see) both with weak lightings and with very brilliant lightings, and secondly to have the aptitude to discriminate between two objects that reflect very nearby intensities between them. To be able to adopt these types of behavior the visual system has two mechanisms: the mechanism of rapid adjustment and the mechanism of local adjustment. Into the first case the retina changes its operative range (range of light intensity) approximately three tenths of second after the change taking place in the light intensity level. In case of the mechanism of local adjustment different parts of the retina adapt to different levels from lighting.

The luminance describes the energy of the stimulus and does not describe the changes of the energy. For this reason the contrast is defined in order to describe the changes of the energy. There are many proposals for the contrast measurement. Basically the contrast can be defined as the change of the relative luminance of the elements of an image. Therefore it corresponds to the difference of luminance that exists between two points of an image. The histogram of the image turns out to be a useful tool to determine the contrast in the image [1].

This work was supported in part by the Spanish Education and Research Council under grant no. TEC2008-04920/MICINN, and by the Andalusia Regional Government under grant no. P08-TIC-03674
In this paper we are going to present a contrast control technique that allows low cost hardware realizations and high processing speed.

The paper is organized as follows. In the next section we will describe images contrast control techniques. Then we will introduce briefly the theoretical concepts of Lukasiewicz logic. Later we will present the proposed techniques. Finally we will consider the circuit design of the basic contrast control operators.

\section{TECHNIQUES OF CONTROL OF CONTRAST}

A definition of contrast is the contrast of Weber and it is commonly used in the context of the lighting. It consists of the difference between two luminance divided by the lowest luminance.

$$
C=\frac{L_{\max }-L_{\min }}{L_{\min }}
$$

Another definition frequently used in photography is that of simple contrast. It is the difference between the brilliant and dark parts of the picture. This definition is not useful for luminance of the real world due to its wide dynamic range and the logarithmic characteristics of the response of the human eye.

$$
C=\frac{L_{\max }}{L_{\min }}
$$

The peak-to-peak contrast or Michelson's contrast measures the relation between spread and the sum of two luminances. This definition is used in signal processing theory for determining the quality of a signal regarding to its noise level. 


$$
C_{M}=\frac{L_{\max }-L_{\min }}{L_{\max }+L_{\min }}
$$

A further type of contrast measure is the variance. It is given by the following expression:

$$
\sigma^{2}=\frac{1}{M N} \sum_{k=1}^{L}(k-\bar{k})^{2} n_{k}
$$

where $\mathrm{M}$ and $\mathrm{N}$ are the dimension of the image, $\mathrm{k}$ is he value of the luminance in the range $[1, L], n_{k}$ is the frequency of the $\mathrm{k}$ luminance level, and $\bar{k}$ is the mean value of the luminance distribution,

$$
\bar{k}=\frac{1}{M N} \sum_{k=1}^{L} k n_{k}
$$

When all the pixels have the same gray level its variance is zero, and when the difference between all the possible pairs of pixels is larger the variance is greater.

On the other hand the values $\left\{\mathrm{p}_{\mathrm{k}}=\mathrm{n}_{\mathrm{k}} / \mathrm{MN} ; \mathrm{k}=1,2, \ldots, \mathrm{L}\right\}$ constitute a probability distribution on the set of the luminance values as $\sum_{k=1}^{L} p_{k}=1$. It is possible to use the entropy as a contrast measure [2]:

$$
H=-\sum_{k=1}^{L} p_{k} \ln p_{k}
$$

When the distribution of luminance tones of the pixels is uniform $\left(p_{k}=1 / L\right)$ then the entropy reaches its maximum value (which is $\ln (\mathrm{L})$ ) which corresponds to an image with maximum contrast. This suggests that a standard measure in the interval $[0,1]$ of the contrast of an image is $\mathrm{H} / \mathrm{ln}(\mathrm{L})$.

Note that entropy is a measure of uncertainty. When it goes zero corresponds to minimum contrast, and for an image with uniform distribution, which corresponds to the maximum contrast, the uncertainty or lack of information is maximum.

Due to the process of digitalization of images the pixels are codified by a limited number of bits. For example, in the case of 8-bit monochrome images supposed to distinguish 256 levels of gray. If the range of variation in the brightness of the image is much smaller than the dynamic range of the camera then the true range of numbers will be much smaller than the full range from 0 to 255 . That is, the image obtained at the output of the sensors of the camera does not have to cover the full range. In many situations the recorded image will have a much smaller range of brightness values. These values can be found in the mid-range (intermediate gray values) or to bright or dark ends of the range.

The visibility of the elements that form an image can be improved by stretching the contrast in order to reassign the values of pixels to fill the entire available range. This means that the pixels are interpolated between the extreme values of the dynamic range.

A usual mechanism of contrast enhancement is to perform a linear interpolation [1], [3], [4], [5]. This technique of linear expansion of the contrast allows to increase the visual discrimination and is useful when the image has luminance variations that allow to distinguish between the elements that comprise it.

There are hardware implementations circuits that perform the contrast control. Thus in [4] describes a circuit implemented in a $0.25 \mu \mathrm{m}$ CMOS technology. The method described in [3] applies in video images and is based on the piecewise linear functions approximation of the cumulative density function (CDF).

Other techniques are based on local transformations of the pixels and are called point operations. The point operations, or point to point functions, require in each step to know the value intensity of a single pixel, to which the desired transformation is applied. After processing the pixel is not needed, therefore this type of operation are called zero memory

Point operations are performed more efficiently with lookup tables (LUTs). The LUTs are simple vector that use the value of the current pixel as an index of the vector. The new value is the vector element stored at that position. The new image is constructed by repeating the process for each pixel. Using LUTs avoid repeated and unnecessary computations. When working with images of, for example, 8 bits only need to calculate 256 values. In this case the size of the image is irrelevant since the value of each pixel of the image is a number between 0 and 255 and the result of lookup table produces another number between 0 and 255. These algorithms can be implemented without using any intermediate memory since the output image can be stored in the same memory space that the input.

One of the non-linear transformations most widely used is the Gaussian transformation that is given by:

$$
g(i . j)=\frac{\phi\left(\frac{f(i, j)-0.5}{\sigma \sqrt{2}}\right)+\left[\frac{0.5}{\sigma \sqrt{2}}\right]}{\phi\left(\frac{0.5}{\sigma \sqrt{2}}\right)}
$$

where the brackets in the expression $[\mathrm{x}]$ represent the floor function of $\mathrm{x}$, and

$$
\phi(x)=\frac{2}{\sqrt{\pi}} \int_{0}^{x} e^{-y^{2}} d y
$$

This transformation increases the contrast of the image making the dark parts darker and the bright part clearer. 


\section{III. ŁUKASIEWICZ LOGIC}

The development of the theoretical concepts of the multivalued logics began in the decade of the $20 \mathrm{~s}$ by Jan Lukasiewicz, who established the generalization of the classic logic to the multi-valued logic. Later, at the end of the 50s, C.C. Chang formalized the multi-valued algebra based on Łukasiewicz logic [6]. A multi-valued Łukasiewicz algebra is a structure $A=(A, \oplus, \otimes, \neg, 0,1)$ that satisfies the following properties:

- $x \oplus(y \oplus z)=(x \oplus y) \oplus z$

- $x \oplus y=y \oplus x$

- $x \oplus 0=x$ and $x \oplus 1=1$

- $\neg 0=1$ and $\neg 1=0$

- $\neg(\neg x \oplus \neg y)=x \otimes y$

- $x \oplus(\neg x \otimes y)=y \oplus(\neg y \otimes x)$

The multi-valued algebra coincides with the Boolean algebra if idempotency happens $(x \oplus x=x)$.

The operator's definitions are:

- bounded-sum: $x \oplus y=\min (1, x+y)$

- bounded-product: $x \otimes y=\max (0, x+y-1)$

- implication: $\neg x=1-x$

On the other hand, the following connectives are useful:

$$
\begin{aligned}
& x \vee y=\max (x, y)=(x \otimes \neg y) \oplus y \\
& x \wedge y=\min (x, y)=(x \oplus \neg y) \otimes y
\end{aligned}
$$

In order to visualize the meaning of the operators, figure 1 shows the graphical representation of the bounded-sum and the bounded-product.

\section{CONTRAST CONTROL BY MEANS OF LUKASIEWICZ OPERATORS}

The application of the operators of Lukasiewicz in an image gives place to a transformation of the distribution of the levels of the pixels. This transformation produces a shift from low levels to high or from high levels to low, ie the application of Lukasiewicz operators most of the gray levels of the image undergo a shift in the histogram

The bounded-sum operator acts as a low-pass filter and performs a shift of the pixels to high levels. This way a clearer image is obtained. Figure 2 shows the effect of applying the bounded-sum to consecutive pixels of the original image. It is

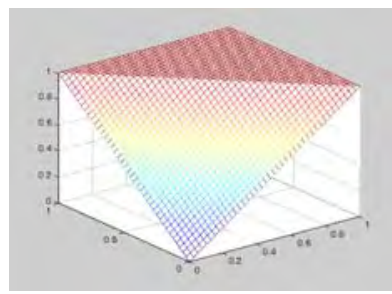

a)

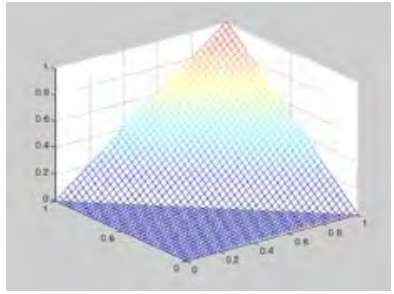

b)
Fig. 1. Surfaces corresponding to the operators

(a) bounded-sum and (b) bounded-product.

possible to observe the displacement of the pixels towards the white by giving a brightness image.

The contrast control using the bounded-sum can be done by introducing an additional parameter that allows to regulate the displacement of the frequency:

$$
x \oplus y \oplus C
$$

where $\mathrm{C}$ is the parameter of control of the contrast. The range of values that can take $C$ (encoded with 8 bits) is in the interval $[-128,127]$. Figure 2 shows the effect of the boundedsum with different values of the control parameter $(\mathrm{C}=0$ and $\mathrm{C}=30$ ).

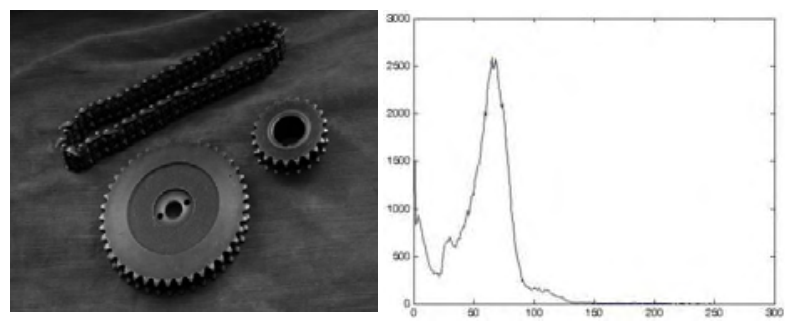

Original image
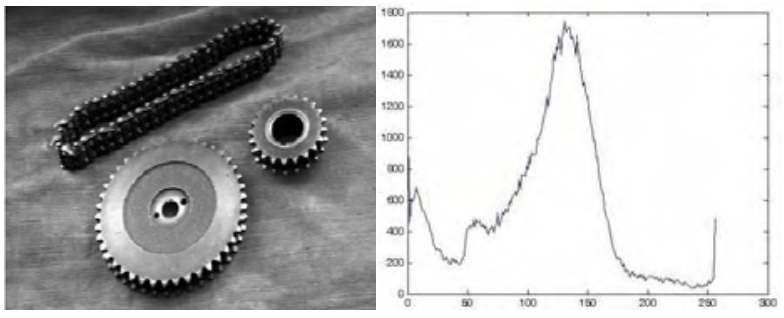

$x \oplus y$

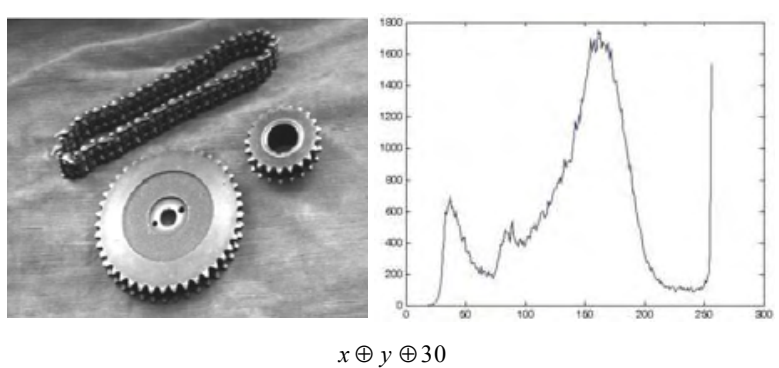

Fig 2. Control of contrast using the bounded-sum and the histogram of the images 


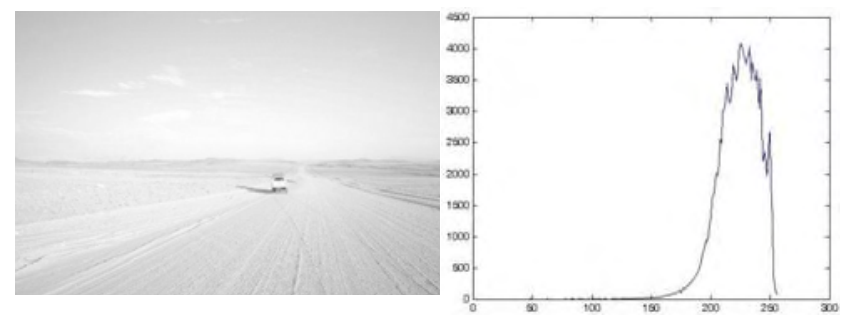

Original image
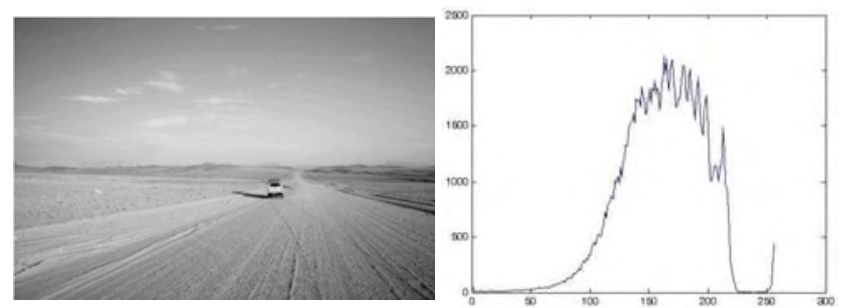

$x \otimes y \otimes(-30)$
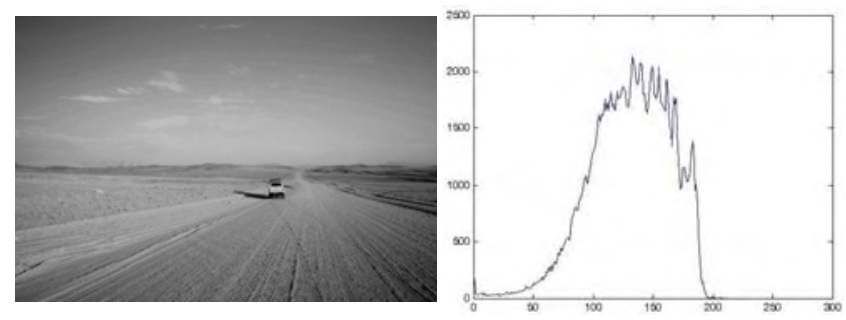

$x \otimes y \otimes(-60)$

Fig. 3. Control of contrast using the bounded-product and the histogram of the images

The complementary operation to the bounded-sum corresponds to the bounded-product. This operator gives place to a displacement of the histogram towards the black. This effect is observed in figure 3 that shows the result of applying the bounded-product and its histogram.

The control of the contrast applying the bounded-product it is realized by means of parameter $\mathrm{C}$ in the following expression:

$$
x \otimes y \otimes C
$$

Figure 3 shows the application of the bounded-product with different values of the control parameter $\mathrm{C}$.

\section{CIRCUIT DESIGN AND IMPLEMENTATION}

The operators that we are going to consider are defined in equations $1-5$. The design of the basic operators circuits will be realized according to two strategies: based on neural networks circuits and based on combinational logic circuits.

In the first implementation strategy we will design the bounded-sum and bounded-product operators by means of neural networks. For it we will follow [7] where is

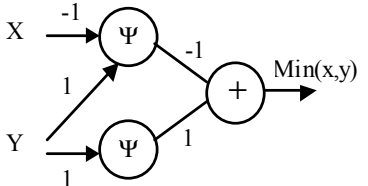

(a)

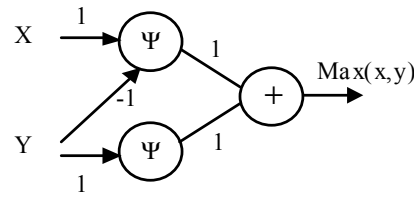

(b)
Fig. 4. Operators $\min (x, y)$ and $\max (x, y)$ realized by means of neural networks

demonstrated that it is possible to represent the neural network corresponding to a combination of propositions of Lukasiewicz's calculus using an activation function $\psi(x)=1 \wedge(x \vee 0)$. The realizations of $\min (\mathrm{x}, \mathrm{y})$ and $\max (\mathrm{x}, \mathrm{y})$ operators as proposed in [8] are shown in figure 4. They consist on one layer networks.

The neuron circuit schematic is shown in figure 5. The inputs and output have been codified with 8 bits. The realization of the bounded product and bounded sum operators is based on the following expressions:

$$
\begin{gathered}
x \oplus y=\min (1, x+y)=1 \wedge(x+y) \\
x \otimes y=\max (0, x+y-1)=0 \vee(x+y-1)
\end{gathered}
$$

It is possible to simplify the design of these operators because only one neuron is needed. Therefore the cost of the realization is determined by the characteristics of the circuit in figure 5 .

The second design strategy is based on combinational logic circuits. Figure 6 shows the design of the boundedproduct and bounded-sum operator. A difference between the neural network realization style and the combinational logic strategy consists in that the latter case is based on descriptions that contain circuit components like comparators, adders, substractors, multiplexers, etc, without establishing a regular structure.

Table 1 shows the different implementations results on Xilinx FPGAs. The cost of the circuits in terms of area is given in slices. Finally, the delay corresponds to the maximum delay between input and output pads.

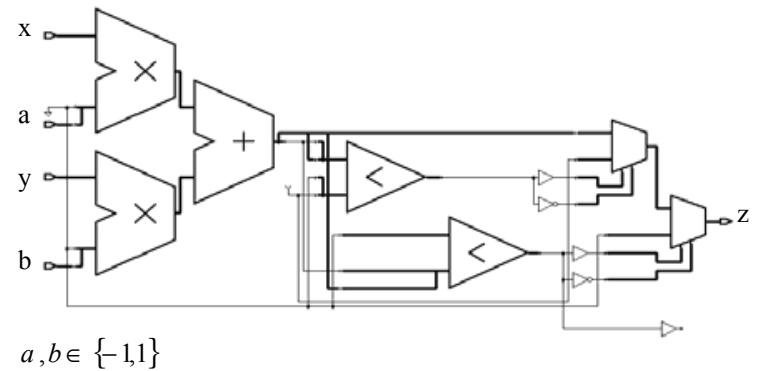

Fig. 5. Circuit schematic of a neuron 


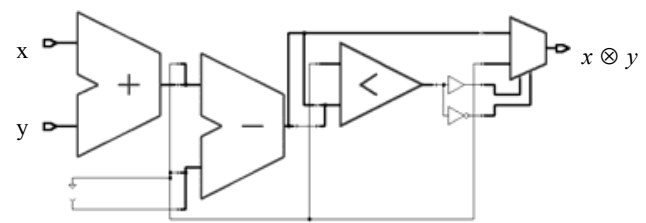

(a)

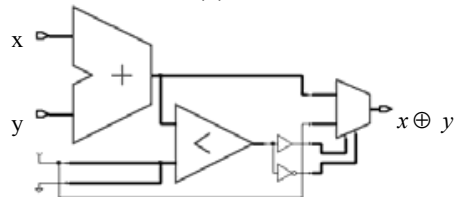

(b)

Fig. 6. (a) bounded product circuit, (b) bounded sum module.

\section{CONCLUSION}

In this paper a technique of control of contrast has been described based on the bounded-sum and bounded-product operators. These operators produce a shift and expansion in the histogram of the image. The resulting circuits are low cost and high processing speed. This makes the proposed contrast control system very suitable to be embedded together with the vision sensor.

\section{REFERENCES}

[1] Z.Y. Chen, B.R. Abidi, D.L. Page, M.A. Abidi, "Gray-Level Grouping (GLG): An Automatic Method for Optimized Image Contrast Enhancement-Part I: The Basic Method", IEEE Transactions on Image Processing, vol. 15, no. 8, pp. 2290-2302, Aug. 2006.
TABLE 1 .

COMP ARATIVE RESUL TS OF THE O PERATORS REALIZATIONS (NOTE: A REA IN SLICES AND DELAY IN NSEC)

\begin{tabular}{|c|c|c|c|c|}
\cline { 2 - 5 } & \multicolumn{2}{c}{ neural network } & \multicolumn{2}{c|}{$\begin{array}{c}\text { combinational } \\
\text { logic }\end{array}$} \\
\hline $\min$ & 8 & 17.63 & 8 & 16.58 \\
\hline $\max$ & 8 & 17.85 & 8 & 16.77 \\
\hline$\bigoplus$ & 15 & 14.84 & 8 & 16,89 \\
\hline$\otimes$ & 13 & 19.64 & 13 & 19.64 \\
\hline
\end{tabular}

[2] Amar Khellaf, Azeddine Beghdadi, and Henri Dupoisot, "Entropic Contrast Enhancement", IEEE Transactions On Medical Imaging, vol. 10, no. 4, pp- 589-592, Dec. 1991.

[3] S.Y. Kim, D. Han, S.J. Choi and J.S. Park, "Image Contrast Enhancement Based on the Piecewise-Linear Approximation of CDF ", IEEE Transactions on Consumer Electronics, Vol. 45, No. 3, pp. 828834, Aug. 1999.

[4] H.C Kim, B.H. Kwon, M.R. Choi "An Image Interpolator with Image Improvement for LCD Controller", IEEE Transactions on Consumer Electronics, vol. 47, no. 2, pp. 263-271, May 2001.

[5] H.H. Cho, C.H. Choi, B.H. Kwon and M.R. Choi: "A Design of Contrast Controller for Image Improvement of Multi-Gray Scale Image ", 2nd IEEE Asia Pacific Conference on ASICs, pp. 131-133, Aug 2000.

[6] A. Di Nola, A. Lettieri, "Formulas of Łukasiewicz's logic represented by hyperplanes," 10th International Fuzzy Systems Association World Congress (IFSA), Istanbul, Turkey, pp. 189-194, 2003.

[7] J.L. Castro, E. Trillas: "The logic of neural networks", Mathware and Soft Computing, vol 5, pp. 23-27, 1998.

[8] P. Amato, A. Di Nola, B. Gerla, "Neural Networks and Rational Lukasiewicz Logic", Proceedings. NAFIPS. Annual Meeting of the North American. pp. 506-510, 2002. 